\title{
Maltrato psicológico.
}

Psychological abuse.

\section{Gómez de Terreros Guardiola1}

\section{RESUMEN}

En la obra de 1989 de Garbarino, Guttman y Seeley, el maltrato psicológico es definido como "un ataque realizado por un adulto sobre el desarrollo de la personalidad y de la competencia social del niño mediante un patrón de conducta psicológicamente destructivo y que se manifiesta mediante cinco formas: rechazar, aislar, aterrorizar, ignorar y corromper", pero es difícil encontrar un trabajo sobre la temática de los malos tratos en general, y sobre el maltrato psicológico en particular, que no haga referencia a los problemas de definición y categorización.

A pesar de que ya van 30 años de investigación que ponen de manifiesto las consecuencias adversas del maltrato 0 abuso emocional en la infancia, las instituciones, en escasas ocasiones, atienden estos casos si no van acompañados de otras formas de maltrato como el abuso sexual o el maltrato físico. Existen una serie de medidas que pueden poner remedio a la situación, pero cuando se observa que no existe una definición consistente y comprensible del maltrato emocional o psicológico, que comprenda tanto al abuso como al abandono o negligencia, cualquier decisión que intente remediar la situación es difícil de asumir.

Una detección y denuncia rápidas de la sospecha de maltrato psicológico a las autoridades pertinentes, así como una intervención precoz, puede prevenir y minimizar las consecuencias de dicho maltrato.

Palabras clave: Maltrato psicológico, maltrato emocional, abuso y abandono emocional, definición.

\section{ABSTRACT}

In 1989, Garbarino, Guttman and Seeley defined psychological abuse as "an attack made by an adult on the development of the personality and the social competence of the child through a repeated pattern of destructive conduct and that is pronounced by means of five forms: to reject, to isolate, to terrify, to ignore and to corrupt, but beside this it is difficult to find a publication on this subject in general, or in psychological abuse in particular, that does not make reference to the problems of definition and categorization.

Despite that already there has been 30 years of investigation that shows the adverse consequences of the emotional abuse or neglect in the infancy, the institutions, in scarce occasions, attend these cases if they do not go accompanied by other forms of sexual or physical abuse. There exists a series of measures that can put remedy to the situation, but when it is observed that there isn't a consistent and understandable definition of the emotional abuse or psychological maltreatment that considers both abuse and neglect, any decision that tries to remedy the situation is difficult to assume. An early detection and accusation of the suspicion of psychological maltreatment to the pertinent institution, as an early intervention, can prevent and minimize the consequences of the abuse.

Key words: Psychological abuse, emotional abuse and neglect, definition.

Cuad Med Forense 2006; 12(43-44):103-116

Correspondencia: Montserrat Gómez de Terreros Guardiola. Departamento de Personalidad, Evaluación y Tratamiento Psicológico. Facultad de Psicología. Calle Camilo José Cela, s/n. 41018 Sevilla. Tfno.: 954556933 E-mail: guardi@us.es.

1 Profesora Titular. Facultad de Psicología de la Universidad de Sevilla. 


\section{INTRODUCCIÓN:}

Maltrato psicológico es un término que se usa, en ocasiones, de manera simultánea a otros términos como maltrato emocional, abuso emocional o abuso psicológico, habiendo sido considerado como "la forma más esquiva y dañina de maltrato en la infancia", representando "el papel central y el factor más destructivo de cualquier forma de maltrato" [I].

La dificultad para identificarlo y definirlo, como se pondrá de manifiesto en este artículo, hace dudar de la precisión de los datos que informan sobre su ocurrencia, sin embargo, es frecuente que se afirme en la literatura que es un problema que sigue en aumento [2]. Los diferentes términos y sus definiciones no son más que la revelación del conflicto para su identificación, información, denuncia o prevención.

Ya en el año 1993 la National Research Council [3] recomendó la importancia de definiciones consensuadas entre los investigadores para cada tipología de maltrato, sobre todo a la vista de la falta de acuerdo social acerca de qué formas de trato a los menores no deben ser permitidas o pueden serle perjudiciales, y qué requisitos deben contemplarse en su identificación (la conducta de los padres, el comportamiento del niño, el contexto social, o la combinación de estas y otras variables) [4].

La mayoría de los autores incluyen esta tipología como una forma más de maltrato. Suelen referirse a ella de forma breve y generalmente utilizando términos y definiciones casi idénticas, probablemente porque están derivadas de los trabajos de Garbarino y sus colaboradores, los cuales se convierten en referencia obligada cuando se estudia el maltrato emocional o psicológico.

Considerando el maltrato y la negligencia en general, y el maltrato psicológico en particular, la premisa principal consiste en que la responsabilidad de que la relación que se establece entre el padre y el niño sea adecuada, recae en el padre. Los niños pueden mostrar un temperamento difícil, ser provocativos o, en algunos casos, manifestar comportamientos que pueden resultar seriamente difíciles de manejar para los padres, tanto que cuestionen su habilidad parental. Sin embargo, si los padres son incapaces de afrontar la situación es su responsabilidad el buscar ayuda. Si, por cualquier razón, son incapaces de solicitarla, es responsabilidad de otros el intervenir en ayuda tanto del niño como de los padres [5].

Cada vez está más aceptado que el abuso y abandono emocional genera un daño en el desarrollo del niño y que este daño se extiende hasta la edad adulta [6], como así lo avalan una serie de estudios que ponen de manifiesto los trastornos emocionales, de la conducta y cognitivos que se manifiestan en la infancia [7], adolescencia [8] y edad adulta [9, 10]. Sin embargo en estos estudios, el abuso emocional ha sido o inferido de las descripciones realizadas de las interacciones padreshijos, o definidos en base a las diferentes definiciones operacionales que se han utilizado en diversos estudios [5].

A pesar de que ya van 30 años de investigación que ponen de manifiesto las consecuencias adversas del maltrato o abuso emocional en la infancia, las instituciones en escasas ocasiones atienden estos casos si no van acompañados de otras formas de maltrato como el abuso sexual o el maltrato físico. Existen una serie de medidas que pueden poner remedio a la situación, pero cuando se observa que no se halla una definición consistente y comprensible del maltrato emocional o psicológico, que comprenda tanto al abuso como al abandono o negligencia, cualquier decisión que intente remediar la situación es difícil de asumir.

Debería establecerse claramente si es la conducta del abusador o el daño que se inflige al menor lo que constituye la prueba relevante. Si el requisito es el daño sufrido, si es esto lo que ha 
de justificar la acción, es probable que no conlleve finalmente intervención alguna, ya que este daño puede no ser evidente hasta años después de que se cometa el abuso. Por el contrario, si se depende únicamente de la conducta del abusador, puede ocurrir que se realicen acciones en las que el niño no haya sufrido daño alguno. En segundo lugar, los profesionales de servicios sociales parecen necesitar de mayor formación así como de mayores recursos. Aunque el abandono psicológico se considera significativamente relacionado con trastornos del comportamiento, más incluso que el maltrato físico o que el abuso sexual, llama la atención la relativamente escasa atención que le prestan los profesionales en la literatura especializada en comparación con otras formas de maltrato y la falta de casos legales publicados en donde únicamente se tenga en consideración el maltrato psicológico. Es como si hubiera un menor interés y preocupación profesional acerca de esta forma de abuso. En tercer lugar, es crítico que cualquier medida hacia el abuso deba ser estructurada de tal manera que evite la re-victimización del niño [2].

La evidencia acerca de que los diferentes tipos de malos tratos tienen diferentes consecuencias sobre los niños, ha aumentado. Consecuencias que afectan al desarrollo emocional y social, al funcionamiento cognitivo y a los resultados escolares, así como a los procesos fisiológicos [ [ I, I 2]. Estos datos que avalan la especificidad de las consecuencias han llevado a los investigadores a estudiar de manera específica dichas secuelas [13]. Sin embargo esta tarea no está bien definida, los altos niveles de co-morbilidad que se da en el maltrato no han permitido el delinear unos claros efectos específicos. La aparición conjunta del abuso y el abandono son más bien una regla que una excepción, y se produce en un rango entre el $46 \%$ y $90 \%$ de los casos y, por tanto, el que se produzca un tipo único de maltrato es raro en la práctica [ $\mathrm{l}$ l].

Se demuestra la importancia de la gravedad y la cronicidad, la importancia del estadio del desarrollo en que se produzca el maltrato, y la aparentemente falta de significación sobre el desarrollo del niño, estando subestimada la cifra de niños que padecen diferentes formas de maltrato [I 4].

Además de las mencionadas dificultades para definir operativamente el abuso emocional, existen otras razones que hacen que sea difícil el informar sobre estos casos. Para algunos especialistas el utilizar los términos abuso o maltrato es excesivo y problemático, especialmente cuando no existe intención de dañar al niño. Existe el dilema entre el deseo de proteger al niño y la falta de disposición a etiquetar o culpabilizar a las personas que tienen la responsabilidad de criar al niño. Sin embargo no debería existir opción, ya que el utilizar estos términos necesariamente obliga a la intervención y protección del niño [5].

Al contrario que en el maltrato físico o en el abuso sexual, el abusador es generalmente el cuidador principal. Ambos padres contribuyen al maltrato y el que no lo inflige se siente incapaz de ofrecer una protección adecuada. Por ello se genera la preocupación de que si la protección del niño requiere la separación de la situación, será a costa de romper un vínculo afectivo, aunque sea un vínculo inadecuado. Si la situación se detecta a tiempo, la intervención es posible sin tener que llegar a la separación. La dificultad es entonces el justificar la intervención temprana, ya que este tipo de maltrato no genera daños físicos en el niño. No se puede retrasar el diagnóstico, pues después los patrones de interacción serán mucho más difíciles de modificar, si acaso habría que cambiar la definición del maltrato emocional para permitir su anterior reconocimiento [5].

Una detección y denuncia rápidas de la sospecha de maltrato psicológico a las autoridades pertinentes, así como una intervención precoz, puede prevenir y minimizar las consecuencias de dicho maltrato [15]. 


\section{DEFINICIÓN:}

Diversos factores distinguen al abuso y abandono emocional de otras formas de maltrato. La tabla I muestra estas diferencias [5]. A pesar de ello, como afirman Jiménez Morago y cols. [16]:"no hay una investigación sobre la temática de los malos tratos que no empiece haciendo referencia a los problemas de definición que se plantean en este ámbito" (pag. 19).

TABLA I: Diferencias entre distintas formas de maltrato. (Glaser, 2002).

\begin{tabular}{|c|c|c|c|}
\hline & Abuso sexual & Maltrato físico & $\begin{array}{c}\text { Maltrato/abandono } \\
\text { emocional }\end{array}$ \\
\hline Acción/interacción & No observable & $\begin{array}{c}\text { Observable o no } \\
\text { observable }\end{array}$ & Observable \\
\hline $\begin{array}{l}\text { Identidad del } \\
\text { abusador }\end{array}$ & Cuestionada & A veces conocida & Conocida \\
\hline $\begin{array}{l}\text { Abusador y cuidador } \\
\text { principal }\end{array}$ & $\begin{array}{l}\text { Generalmente son } \\
\text { personas diferentes }\end{array}$ & $\begin{array}{c}\text { Son la misma persona } \\
\text { o diferente }\end{array}$ & Es la misma persona \\
\hline Definición/evidencia & Malos tratos & $\begin{array}{l}\text { Señales de daño en el } \\
\text { niño }\end{array}$ & $\begin{array}{l}\text { Malos tratos del } \\
\text { cuidador y señales de } \\
\text { daño en el niño }\end{array}$ \\
\hline $\begin{array}{l}\text { Indicación de } \\
\text { protección inmediata }\end{array}$ & $\mathrm{Si}$ & Normalmente & Rara vez \\
\hline
\end{tabular}

Es interesante que Glaser [5] menciona una serie de trabajos que ponen de manifiesto cómo, a pesar de las dificultades para definir el maltrato emocional, cuando se indaga entre el público en general (mediante la opinión de ciudadanos), existe un acuerdo muy superior entre lo que consideran abusos emocionales o incluso "conductas inaceptables", que en el acuerdo, por ejemplo, en el reconocimiento de las conductas que constituyen abuso sexual o maltrato físico.

Ha existido bastante debate en cuanto a si la definición de maltrato y abandono emocional debía hacer referencia a la conducta del que maltrata o a las consecuencias sobre el niño, o si ambos eran necesarios para su diagnóstico. En 199| [17] se afirma que para la definición del maltrato emocional la evidencia debe estar en la conducta de maltrato más que en el daño infligido.

Los siguientes criterios constituyen una completa definición de maltrato y abandono emocional (o psicológico) [5]:

- Maltrato y abuso emocional describe una relación entre el padre y el hijo (más que una serie o repetición de acciones que ocurren en dicha relación);

- Estas interacciones impregnan o caracterizan la relación;

- Las interacciones son real o potencialmente dañinas pues causan alteraciones en la salud o en el desarrollo del niño.;

- Incluye tanto la acción como la omisión; y

- El contacto físico no es condición indispensable para el maltrato o abandono emocional.

Es tal el número de comportamientos que quedarín incluidos bajo esta definición que no se puede hacer un listado exhaustivo de ellos [5], aunque la American Professional Society on the Abuse con Children [18] (APSAC) realiza una propuesta que veremos posteriormente. Sin embargo, 
no existe una evidencia empírica que sustente este listado, además no son comportamientos excluyentes o los ejemplos que se aportan no encajan, necesariamente, en determinadas subcategorías.

Se ha desarrollado una estructura alternativa, no basada en la conducta de los padres o en las interacciones que manifiestan con los hijos, sino que se categorizan las formas de maltrato y abandono emocional en función de los elementos que comprometen al ser psicológico del niño o a su ser infantil. Un niño es [4]:

- Una persona que existe;

- Que tiene sus propios atributos;

- Que por definición es vulnerable, dependiente y está inmerso en un rápido desarrollo;

- Un individuo que tiene y experimenta sentimientos, pensamientos y percepciones; y

- Un ser social que cada vez más interactuará y se comunicará dentro de su contexto social.

Cada uno de estos aspectos debe ser reconocido, respetado y valorado por los cuidadores principales o por los padres. La violación o el fracaso hacia el respeto de cualquiera de estos elementos del ser del niño constituyen abuso o abandono emocional.

\section{I.- Semejanzas y diferencias entre abuso/maltrato emocional y psicológico.}

Algunos autores diferencian entre abuso emocional, abuso psicológico y negligencia psicológica; en algunas clasificaciones se habla de maltrato y abandono emocional, y en otras nos encontramos a todas ellas bajo el epígrafe de Maltrato Psicológico.

El abuso emocional es un término definido de manera diferente en los trabajos consultados, definiciones que aparecen en la literatura expresan: "hostilidad, frialdad persistente o rechazo que obstaculiza el normal desarrollo físico o emocional del niño o que genera en él problemas de conducta" [19] "el efecto que, sobre la conducta del niño y sobre su desarrollo emocional, origina un maltrato o rechazo emocional severo y persistente. Todo abuso conlleva alguna forma de maltrato o rechazo emocional; esta tipología debería ser usada cuando es la única forma de maltrato o se usa de manera predominante frente a otras formas del mismo" [20] y "la respuesta repetitiva e inapropiada que se ofrece al niño ante sus experiencias emocionales y las expresiones que las acompañan. El abuso emocional inflige repetidamente daño emocional en el niño (por ejemplo miedo, humillación, ansiedad o desesperanza)".

Algunos autores recomiendan la distinción entre ambos términos, insistiendo en que el maltrato psicológico afecta a las facultades mentales (especialmente cognitivas) y a las morales [2 I]. Sin embargo Glaser [5], en uno de los trabajos más interesantes publicados recientemente, considera inútil esta distinción porque los aspectos cognitivos y emocionales no son independientes.

La superposición que se observa en las definiciones hace difícil diferenciar entre abuso o maltrato emocional o psicológico. Debido, entre otras cuestiones a que ambos términos ponen el énfasis en lo repetitivo de este comportamiento y en las consecuencias que genera sobre el comportamiento del niño.

En la obra de 1989 de Garbarino, Guttman y Seeley [I], el maltrato psicológico es definido como "un ataque realizado por un adulto sobre el desarrollo de la personalidad y de la competencia social del niño mediante un patrón de conducta psicológicamente destructivo y que se manifiesta mediante cinco formas: rechazar, aislar, aterrorizar, ignorar y corromper" (pág.8). En la tabla 2 pueden observarse las definiciones y las conductas o manifestaciones que las provocan. 
TABLA II: Definición y manifestaciones de las distintas formas de maltrato psicológico. Garbarino, Guttman y Seeley (1989).

\begin{tabular}{|c|c|c|}
\hline Formas & Definición & Manifestaciones \\
\hline RECHAZAR & $\begin{array}{l}\text { Negarse a admitir la } \\
\text { legitimidad e importancia } \\
\text { de las necesidades del } \\
\text { niño. }\end{array}$ & $\begin{array}{ll}\text { - } & \text { Rechazar las iniciativas de apego del } \\
\text { niño. } & \\
\text { - } & \text { Excluir activamente al niño de las } \\
& \text { actividades familiares. } \\
\text { - } & \text { Realizar valoraciones negativas } \\
& \text { constantes. } \\
\end{array}$ \\
\hline AISLAR & $\begin{array}{l}\text { Privar al niño de } \\
\text { oportunidades para } \\
\text { entablar relaciones } \\
\text { sociales. }\end{array}$ & $\begin{array}{ll} & \text { Negar la interacción con compañeros } \\
\text { y adultos. } \\
\text { - } & \text { Impedir relaciones sociales }\end{array}$ \\
\hline ATERRORIZAR & $\begin{array}{l}\text { Amenazar al niño de } \\
\text { forma siniestra, } \\
\text { haciéndole creer que el } \\
\text { mundo es caprichoso y } \\
\text { hostil. }\end{array}$ & $\begin{array}{ll}\text { - } & \text { Utilización del miedo como } \\
\text { disciplina. } \\
\text { - } \\
\text { Amenazas a la sensación de } \\
\text { seguridad del niño. } \\
\text { - } \\
\text { Amenazas dramáticas, misteriosas, } \\
\text { extremas. }\end{array}$ \\
\hline IGNORAR & $\begin{array}{l}\text { Privar al niño de la } \\
\text { estimulación necesaria, } \\
\text { limitando su crecimiento } \\
\text { emocional y su desarrollo } \\
\text { intelectual. }\end{array}$ & $\begin{array}{ll}\text { - } & \text { Falta de atención al niño. } \\
\text { - } & \text { Frialdad y falta de afecto. } \\
\text { - } & \text { Faltas de protección ante sus } \\
& \text { demandas de ayuda. }\end{array}$ \\
\hline CORROMPER & $\begin{array}{l}\text { Favorecer conductas que } \\
\text { impiden la normal } \\
\text { integración del niño en la } \\
\text { sociedad, reforzar pautas } \\
\text { de conducta antisocial. } \\
\end{array}$ & 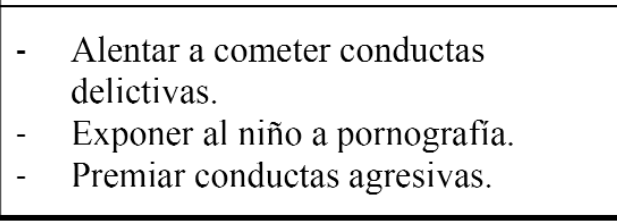 \\
\hline
\end{tabular}

Conviene aclarar que no todos los autores reconocen estos subtipos dentro del maltrato psicológico y, por ejemplo Arraubarrena y de Paúl [22] y Jiménez Morago y cols. [16], consideran la corrupción como un tipo de maltrato fuera de la categoría anterior.

La tabla 3 muestra otras definiciones que continúan utilizándose en la literatura. La cuestión que se plantea Loue [2] es: "si no se ha podido definir icómo se va a abordar?" (pag. 3।2).

\section{2.- Sobre la negligencia o abandono emocional.}

El comportamiento negligente es la conducta realizada por un cuidador que constituye un fracaso en la acción que, según la cultura de una sociedad, es necesaria para satisfacer las necesidades de un niño y que es responsabilidad del cuidador el proporcionarla [23].

Sobre esta definición el autor profundiza en la significación de algunos términos. "Conducta realizada por un cuidador" intentando diferenciar el comportamiento negligente tanto de las posibles causas del mismo (por ejemplo, la enfermedad mental del cuidador), como de sus posibles efectos (daño físico que puede producir), sin por ello restar importancia a ambos aspectos mencionados (causas y consecuencias). 
TABLA III: Definiciones de abuso/maltrato emocional o psicológico.

\begin{tabular}{|c|c|c|}
\hline Autor/as & Año & Definición \\
\hline $\begin{array}{l}\text { Hart, Germain y } \\
\text { Brassard }[45]\end{array}$ & 1983 & $\begin{array}{l}\text { Abuso emocional al niño, como única y central forma de maltrato } \\
\text { consiste en acciones y omisiones en base al juicio tanto de la } \\
\text { comunidad como de los profesionales expertos como psicológicamente } \\
\text { perjudiciales. Dichos actos han de ser cometidos por figuras parentales } \\
\text { que estando en distinta posición de poder hacen al niño vulnerable. } \\
\text { Tales hechos dañan inmediata y en última instancia el funcionamiento } \\
\text { cognitivo, afectivo, social, psicológico y comportamental del niño. }\end{array}$ \\
\hline $\begin{array}{l}\text { Arruabarrena, de } \\
\text { Paúl [22] }\end{array}$ & 1994 & $\begin{array}{l}\text { El maltrato emocional. Hostilidad verbal crónica en forma de insulto, } \\
\text { desprecio, crítica o amenaza de abandono y constante bloqueo de las } \\
\text { iniciativas de interacción infantiles (desde la evitación hasta el } \\
\text { encierro o confinamiento) por parte de cualquier miembro adulto del } \\
\text { grupo familiar. Abandono emocional. Falta persistente de respuesta a } \\
\text { las senales (llanto, sonrisa), expresiones emocionales y conductuales } \\
\text { procuradoras de proximidad e interacción iniciadas por el niño y la } \\
\text { falta de iniciativa de interacción y contacto, por parte de una figura } \\
\text { adulta estable. }\end{array}$ \\
\hline $\begin{array}{l}\text { American } \\
\text { Professional } \\
\text { Society on the } \\
\text { Abuse of } \\
\text { Children [18] }\end{array}$ & 1995 & $\begin{array}{l}\text { Maltrato psicológico significa un patrón repetitivo de la conducta del } \\
\text { cuidador, o un incidente extremo que transmita al niño que es } \\
\text { despreciable, raro, que no es amado, o que peligra su vida si no realiza } \\
\text { determinadas demandas. Aquí se incluyen como ejemplos: } \\
\text { Cuando se dan de manera grave y continuada, los siguientes } \\
\text { comportamientos constituyen maltrato psicológico: } \\
\text { 1) Rechazar (minusvalorando, degradando, avergonzando o } \\
\text { ridiculizando al niño; discriminando mediante la crítica o el } \\
\text { castigo, humillando al niño en público). } \\
\text { 2) Aterrorizar (mediante acciones que pongan en peligro su vida; no } \\
\text { haciéndole sentirse a salvo, creándole falsas expectativas mediante } \\
\text { amenazas de peligro, de pérdida o de dolor si no cumple; } \\
\text { amenazando o actuando violentamente sobre aquellos objetos o } \\
\text { personas que el niño quiere). } \\
\text { 3) Explotar o corromper fomentando en el niño el desarrollo de } \\
\text { conductas inapropiadas (imitando, permitiendo o fomentando } \\
\text { conductas antisociales o inapropiadas para su estadio del } \\
\text { desarrollo; fomentando u obligando a abandonar conductas } \\
\text { autónomas apropiadas para su desarrollo, restringiendo o } \\
\text { interfiriendo en el desarrollo cognitivo del niño). } \\
\text { 4) Negar respuestas emocionales (ignorando o negándose a mostrar } \\
\text { afecto, cuidado y amor al niño). } \\
\text { 5) Rechazar (evitando o alejando al niño). } \\
\text { 6) Aislar (recluyendo, estableciendo limitaciones poco razonables a } \\
\text { la libertad de movimientos o a las interacciones sociales). } \\
\text { 7) Ejercer la paternidad de manera inconsistente o poco fiable } \\
\text { (mediante demandas contradictorias y ambivalentes). } \\
\text { 8) Desatender la salud mental, fisica y las necesidades educativas } \\
\text { (ignorando, impidiendo o no llevando a cabo los tratamientos o } \\
\text { acudiendo a los servicios que atienden las necesidades o } \\
\text { dificultades emocionales, comportamentales, físicas o educativas). } \\
\text { 9) Hacer testigo al niño de íntima violencia con el cónyuge (violencia } \\
\text { doméstica). }\end{array}$ \\
\hline
\end{tabular}


TABLA III continuación: Definiciones de abuso/maltrato emocional o psicológico.

\begin{tabular}{|c|c|c|}
\hline $\begin{array}{l}\text { Jiménez } \\
\text { Morago, Moreno } \\
\text { Rodríguez, } \\
\text { Oliva Delgado, } \\
\text { Palacios } \\
\text { González y } \\
\text { Saldaña Sage } \\
{[16]}\end{array}$ & 1995 & $\begin{array}{l}\text { Maltrato emocional. No se toman en consideración las necesidades } \\
\text { psicológicas del niño o de la niña, particularmente las que tienen que } \\
\text { ver con las relaciones interpersonales y con la autoestima. Indicadores } \\
\text { serían el rechazar al niño, aterrorizarle, privarle de relaciones sociales, } \\
\text { insultarle, ridiculizarle, ignorar sus necesidades emocionales y de } \\
\text { estimulación, notable frialdad afectiva. Su aparición aislada no es } \\
\text { suficiente para determinar la existencia de este tipo de maltrato. }\end{array}$ \\
\hline Christensen [46] & 1999 & $\begin{array}{l}\text { Abuso emocional: El adulto de manera sistemática expone al niño a } \\
\text { insultos, reclusión, amenazas y menosprecio. Abandono emocional } \\
\text { pasivo: el niño es sometido a negligencia o a falta de estimulación } \\
\text { debido a la incapacidad de los padres para proporcionar seguridad, } \\
\text { cuidado y amor, por ejemplo debido a enfermedad mental, abuso de } \\
\text { sustancias (alcohol, medicinas, narcóticos), problemas sociales, etc. }\end{array}$ \\
\hline $\begin{array}{l}\text { American } \\
\text { Academy of } \\
\text { Pediatrics [15] }\end{array}$ & $\begin{array}{l}2000 \\
2002\end{array}$ & $\begin{array}{l}\text { Maltrato psicológico es un patrón repetitivo de interacciones } \\
\text { perjudiciales entre un/los padre/s y el niño de tal manera que se } \\
\text { convierten en la forma habitual de relación. En algunas situaciones el } \\
\text { patrón es crónico y dominante; en otras, solo ocurre bajo los efectos } \\
\text { del alcohol o desencadenados por otros factores potenciales. De } \\
\text { manera ocasional puede producirse ante la ocurrencia de un único } \\
\text { hecho particularmente doloroso o nefasto. El maltrato psicológico } \\
\text { ocurre cuando una persona transmite a un niño que es despreciable, } \\
\text { que está lleno de defectos, que no es amado, que está en peligro, o que } \\
\text { sólo sirve para atender sus necesidades. El autor de este tipo de } \\
\text { maltrato suele despreciar, aterrorizar, aislar, ignorar o impedir la } \\
\text { socialización del niño. }\end{array}$ \\
\hline Glasser [5] & 2002 & $\begin{array}{l}\text { Maltrato y abandono emocional describe la relación entre el padre y } \\
\text { el niño (más que un hecho o una serie de ellos que ocurre en la } \\
\text { relación padre-hijo); unas interacciones preocupantes que impregnan o } \\
\text { caracterizan la relación; unas interacciones que causan o pueden } \\
\text { causar una alteración en el desarrollo o estado psicológico o emocional } \\
\text { del niño; El abuso y abandono emocional incluyen tanto la acción } \\
\text { como la omisión y no requieren del contacto físico. }\end{array}$ \\
\hline
\end{tabular}

El maltrato psicológico es difícil de definir operativamente [24] mientras que no lo es la negligencia o abandono emocional [25] que, sin embargo, muestra su dificultad a la hora de demostrar sus consecuencias sobre el desarrollo del niño [26].

\section{3.- Categorías del abuso y abandono emocional.}

Las siguientes cinco categorías se incluyen en una completa definición del abuso y abandono emocional [27]:

a. Falta de disposición emocional, falta de respuesta y abandono; incluye insensibilidad parental. El cuidador principal está normalmente preocupado por sus propias dificultades tales como su salud mental, sus problemas con las drogas o sus insoportables compromisos laborales. Son por tanto incapaces de responder a las necesidades emocionales de los hijos sin capacidad de proporcionarles alternativas adecuadas.

b. Atribuciones negativas o pobres hacia el niño; muestras de hostilidad, de desprecio y rechazo hacia el niño que es percibido como merecedor de ellas. Algunos niños crecen y actúan conforme a estas atribuciones que se han depositado en ellos. 
c. Interacciones inapropiadas o inconsistentes respecto al desarrollo del niño; expectativas por debajo de las capacidades que el niño posee por su desarrollo; sobreprotección y limitación de la exploración y del aprendizaje; exposiciones hacia hechos o interacciones que le pueden confundir o traumatizar (exposición a violencia doméstica, por ejemplo); Las interacciones con los hijos, aunque dañinas, son irreflexivas y equivocadas más que con intención de causar daño.

d. Fracaso en el reconocimiento de la individualidad del niño y de los límites psicológicos; utilizando al niño para satisfacer las necesidades de los padres; falta de habilidad para distinguir entre la realidad del niño y las creencias y deseos del niño; esta categoría se aprecia cuando existen conflictos en el divorcio de los padres.

e. Fracaso para fomentar la adaptación social del niño; fomentando la falta de socialización (incluyendo la corrupción), el abandono psicológico (fracaso para proporcionar una estimulación cognitiva adecuada y / u oportunidades para el aprendizaje); incluye tanto la acción como la omisión, incluye el aislamiento del niño e involucrarle en actividades criminales.

Una clasificación debe ser útil tanto para los clínicos como para los investigadores. Quizá el escaso éxito de los tratamientos en el maltrato psicológico sea debido a que las intervenciones que se realizan no son específicas. Es decir, las distintas categorías requieren de diferentes tratamientos. Por otra parte la fiabilidad inter-jueces y la independencia de las categorías deben demostrar su utilidad [5].

\section{INCIDENCIA Y CAUSAS DEL MALTRATO PSICOLÓGICO:}

Igual que ocurre con otras formas de maltrato, la verdadera incidencia y prevalencia de esta tipología son desconocidas. Cuando se da como única forma de maltrato tiene el índice más bajo de confirmación de las diferentes tipologías [15]. En el Informe Nacional de Maltrato en la Infancia realizado en 1997 en Estados Unidos en maltrato psicológico o emocional fue denunciado en un 6. $1 \%$ de los casos [28].

En 1996 el 15\% de todos los registros de malos tratos en Inglaterra fueron relativos al maltrato psicológico [29]. En 2000 ya se recogen cifras del $18 \%$ de los registros realizados por el Children's Bureau [30]. Esto significa que aunque se recoge esta forma de maltrato y se reconoce la necesidad de intervención, no se tiene en cuenta que el resto de niños maltratados probablemente también lo sean psicológicamente [5].

Estudios españoles ponen de manifiesto que en el País Vasco en una investigación realizada por De Paúl en la provincia de Guipúzcoa, el maltrato y abandono emocional se daban en un 4\% de los casos [16]. En Cataluña se ofrecen cifras mucho mayores en 1991, siendo el maltrato psicológico el segundo más frecuentemente detectado, en un 43,6\% de los casos, y tras la negligencia o abandono [3 I]. En 1992, un informe para la Diputación General de Aragón [32] revela que en la ciudad de Zaragoza se estimaba que un 32,2\% de los niños maltratados sufrían maltrato psicológico, segundo de los tipos de maltrato más frecuente después del abandono. En la Comunidad de Madrid [33] si se agrupa el maltrato y el abandono emocional encontrándose una cifra de 29,2\% de los casos que lo padecen.

En Andalucía, en 1995, se publica el primer trabajo sobre incidencia del maltrato en toda la comunidad a través de la revisión de casos mediante expedientes de las Delegaciones Provinciales de la Consejería de Trabajo y Asuntos Sociales, informes de los Servicios Sociales Comunitarios, colegios públicos y privados y dispositivos sanitarios, todos ellos referidos al año 1992. El primer 
dato de interés es la afirmación de que el maltrato infantil afecta aproximadamente a I 5 de cada mil niños andaluces. El maltrato emocional se produjo en un 45,5\% de los casos, siendo la tipología más frecuente tras el abandono. Al igual que en otros estudios, este reconoce que aproximadamente la mitad de los niños sufre más de una forma de maltrato. Ambas formas de maltrato más frecuentes se dan conjuntamente en un I4,2\% de los casos y el maltrato emocional, como única forma, en un $9,1 \%$ de los casos estudiados. No existen diferencias significativas en este estudio respecto al sexo de los niños maltratados emocionalmente. Con respecto a las edades de estos niños, existe un incremento claro a medida que la edad aumenta, manteniéndose aproximadamente en un 7\% desde los siete a los trece años. A partir de esta edad, el porcentaje decae bruscamente [16].

En este estudio se afirma que en el maltrato psicológico son los padres los responsables de su aparición, conjunta o separadamente. Solo en un $6 \%$ de los casos lo producen otros familiares y en un $0,1 \%$ otras personas.

En cuanto al maltrato en niños con deficiencias en nuestro país un estudio [34] refleja una incidencia en Castilla La Mancha del I5,5\% frente al I,5\% de los menores sin discapacidad. El gráfico I muestra la tipología de malos tratos sufridos por estos niños. Aunque no se ha hecho una revisión de este tema específicamente, quisiera poner de manifiesto este problema.

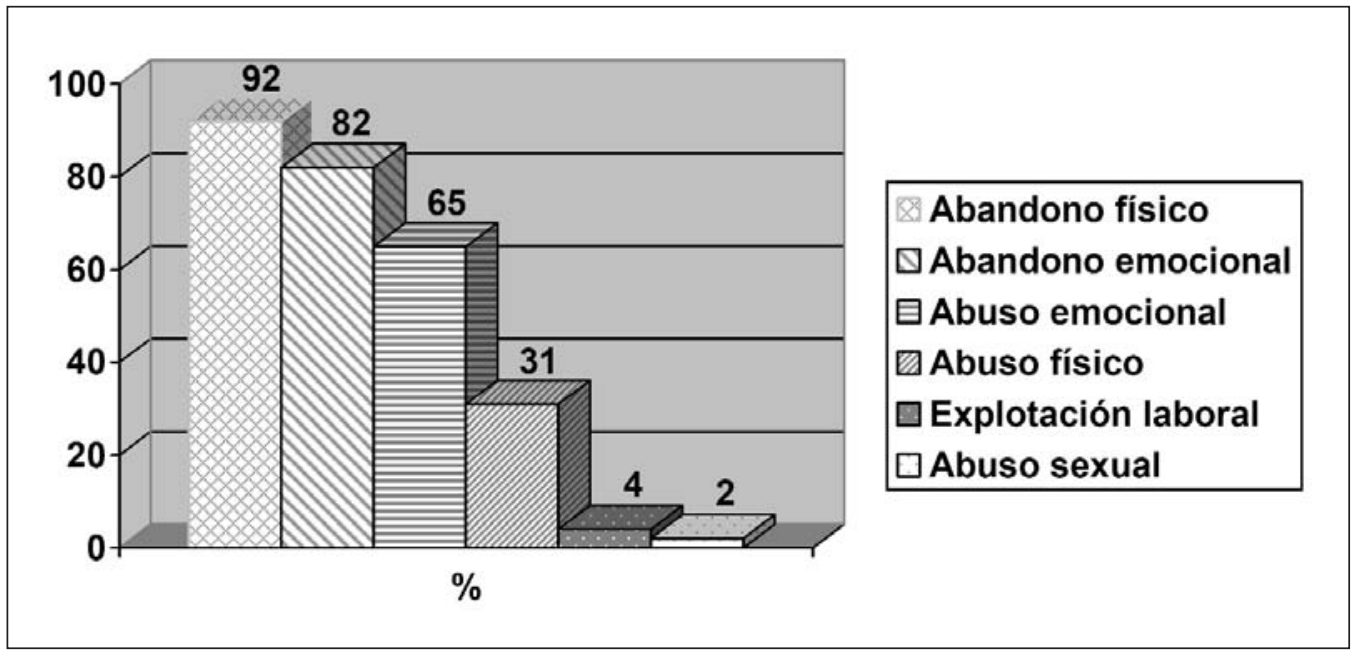

Gráfico 1. Prevalencia de los diferentes tipos de malos tratos en menores con retraso mental. (Tomado de Simón Rueda y cols. y adaptado de Verdugo y cols.)

Las características de los padres incluyeron pobres habilidades parentales, abuso de sustancias, depresión, intentos de suicidio u otros trastornos psicológicos, baja autoestima, pobres habilidades sociales, estilo educativo autoritario, falta de empatía, estrés social, violencia doméstica y disfunción familiar[35]. Un número importante de estudios ponen de manifiesto que un trastorno afectivo en la madre y/o el abuso de sustancias correlaciona significativamente con interacciones verbales agresivas entre los padres y sus hijos [36].

En el estudio de Jiménez y cols [16], se afirma que existen diferentes características entre los padres que llevan a cabo diferentes tipos de malos tratos. Concretamente, los padres que maltratan emocionalmente presentan con mayor frecuencia antecedentes psiquiátricos y con menor frecuencia antecedentes de delincuencia, alcoholismo y drogadicción o prostitución. Existen, además, fuertes desavenencias conyugales en un $56,2 \%$ de estas familias, cifra mayor que la que se 
muestra en otras tipologías. También se producen dificultades económicas y desempleo en cifras muy altas (46,4\% y $62,7 \%$ de los casos), aunque estas características familiares no se diferencian de las familias en donde se produce Maltrato Físico o Abandono.

Finalmente, insistir en que niños en riesgo son aquellos cuyos padres están inmersos en un divorcio contencioso, niños no queridos o no planificados, niños cuyos padres son inexpertos o poco hábiles en su paternidad, padres con problemas de abuso de sustancias, padres que abusan de animales, niños expuestos a violencia doméstica, niños aislados socialmente o discapacitados [37].

\section{CONSECUENCIAS DEL MALTRATO PSICOLÓGICO:}

Es frecuente que coexistan diferentes tipos de maltrato y abandono, por ejemplo, Claussen y Crittenden [38] encuentran que un 90\% de los niños físicamente maltratados o abandonados, según su estudio, habían sido maltratados psicológicamente. Además hallaron que este tipo de maltrato predecía mejor posteriores alteraciones del desarrollo del niño que la gravedad del maltrato físico. Tras el daño físico que puede resultar del maltrato o abandono, la mayoría de las secuelas afectan al desarrollo psicológico, emocional y de la conducta, lo que implica que esta forma de maltrato debe ser atendida como un mediador del dolor causado por otras formas de maltrato $[39,40]$.

Probablemente, cuando el maltrato psicológico ocurre como única forma de maltrato, tenga un efecto más adverso en el funcionamiento psicológico del niño y posterior adulto que incluso el maltrato físico, sobre todo respecto a medidas de depresión y autoestima [38], agresividad, delincuencia o problemas en las relaciones interpersonales [4I].

Seguramente tendrá múltiples consecuencias a largo plazo para el niño. Consecuencias que pueden variar en función de la edad del niño [42,43], ya que un patrón cronificado de maltrato psicológico destruye el sentido de y la seguridad en uno mismo. Esto conlleva las siguientes consecuencias [6]:

I) A nivel de pensamientos, como sentimientos de baja autoestima (incluyendo las conductas asociadas al mismo), visión negativa de la vida, síntomas de ansiedad y depresión, así como ideas de suicidio.

2) Sobre la salud emocional, como inestabilidad emocional, personalidad borderline o límite, falta de respuestas emocionales apropiadas, problemas de control del impulso, ira, conductas autolesivas, trastornos de la alimentación y abuso de sustancias.

3) Sobre las habilidades sociales, incluyendo conducta antisocial, problemas de vinculación afectiva, competencia social limitada, falta de simpatía y empatía, aislamiento social, dificultad para ajustarse a las normas, mala adaptación sexual, dependencia, agresividad y violencia y delincuencia o criminalidad.

4) Sobre el aprendizaje, bajo rendimiento escolar, dificultades de aprendizaje y dificultades en el desarrollo moral.

5) Sobre la salud física, fallos de medrar, quejas somáticas, un estado pobre de salud y alta mortalidad.

Estos síntomas son similares a los que se observan en niños que están sometidos a violencia doméstica, donde se les aterroriza, explota o corrompe, lo cual aumenta la conducta desobediente y los síntomas de depresión, ansiedad y agresividad [44].

Autores españoles [16] consideran "evidentes" las características de los niños maltratados psicológicamente. Estos niños suelen presentar más frecuentemente trastornos emocionales como miedo (23,2\%), agresividad (29,4\%) e inestabilidad emocional (51,3\%). La información que se halla 
de estos casos es generalmente más detallada debido a que suelen ser diagnosticados por unidades especializadas de Salud Mental.

En la tabla 4 se proponen una serie de consecuencias o indicadores de maltrato emocional [33] que complementan este apartado y que han sido expuestas por autores españoles.

TABLA IV: Indicadores de maltrato emocional en los niños. (Simón Rueda, López Taboada, Linaza Iglesias, 2000).

\begin{tabular}{|c|c|}
\hline INDICADORES FÍSICOS & $\begin{array}{l}\text { - Retraso en el crecimiento } \\
\text { - Enfermedades psicosomáticas } \\
\text { - Accidentes frecuentes }\end{array}$ \\
\hline $\begin{array}{l}\text { INDICADORES } \\
\text { CONDUCTUALES }\end{array}$ & $\begin{array}{l}\text { - Dificultades en su desarrollo evolutivo: lenguaje, inteligencia, } \\
\text { psicomotricidad, socialización... } \\
\text { - Muestras de recelo, permisividad, ansiedad, depresión, } \\
\text { preocupación... } \\
\text { - Signos de trastornos emocionales como movimientos rítmicos } \\
\text { repetitivos, ausencia de comunicación... } \\
\text { - Trastornos conductuales como retraimiento y conducta antisocial. } \\
\text { - Trastornos conductuales como pasividad o conducta agresiva } \\
\text { extrema. } \\
\text { - Excesiva rigidez y conformismo. } \\
\text { - Excesiva preocupación por complacer a las figuras de autoridad. } \\
\text { - Conductas adultas inapropiadas o excesivamente infantiles. } \\
\text { - Pobre autoestima. } \\
\text { - Fugas de casa. } \\
\text { - Trastornos del control de esfinteres, trastornos del sueño. } \\
\text { - Psicopatías... }\end{array}$ \\
\hline
\end{tabular}

En definitiva, desde los primeros trabajos que se publicaron en España sobre el maltrato psicológico (incluyendo el abuso y el abandono emocional) a la actualidad, se ha avanzado mucho en su definición, diagnóstico, evaluación e intervención. Queda mucho por hacer, pero las instituciones están muy sensibilizadas, lo que hará progresar enormemente el estudio de esta forma de maltrato.

\section{BIBLIOGRAFÍA:}

I. Garbarino J, Guttman E, Seeley JW: The psychologically battered child. Strategies for identification, assessment and intervention. $4^{\mathrm{a}}$ ed. Jossey-Bass Inc., Publishers. San Francisco, 1989.

2. Loue S: Redefining the emotional and psychological abuse and maltreatment of children. Legal implications. Journal of Legal Medicine 2005; 26: 3II-337.

3. National Research Council (NRC): Understanding child abuse and neglect. National Academy Press. Washington, 1993.

4. Barnett D, Manley JT, Ciccetti D: Defining child maltreatment: The interface between policy and research. En Ciccetti D, Toth SL: Child abuse, child development and social policy. I ed. Ablex. Norwood, 1993. pp. 7-73.

5. Glaser D: Emotional Abuse and Neglect (Psychological Maltreatment): A Conceptual Framework. Child Abuse \& Neglect 2002; 26: 697-702.

6. Hart S, Binggeli N, Brassard M: Evidence of the effects of psychological maltreatment. Journal of Emotional Abuse 1998; I: 27-58. 
7. Erickson M, Egeland B, Pianta R: The effects of maltreatment on the development of young children. En Ciccetti D, Carlson V: Child maltreatment: Theory and research on the causes and consequences of chil abuse and neglect. Cambridge University Press. Cambridge,1989. pp. 647-684.

8. Herrenkohl RC, Herrenkohl EC, Egolf B, Wu P: The developmental consequences of child abuse: the Lehigh longitudinal study. En Starr R, Wolfe D: The effects of child abuse and neglect, issues and research. Guilford Publications, New York, 1991. pp. 57-80.

9. Briere J, Runtz M: Differential adult symptomatology associated with three types of child abuse histories. Child Abuse \& Neglect 1990; 14: 357-364.

10. Mullen P, Martin J, Anderson J, Romans S, Herbison G: The longterm impact of the psysical, emotional, and sexual abuse of children: a community study. Child Abuse \& Neglect 1996; 20: 7-21.

II. Lau AS, Leeb RT, English D, Graham JC, Briggs EC, Brody KE, Marshall JM: What's in a name? A comparison of methods for classifying predominant type of maltreatment. Child Abuse \& Neglect 2005; 29: 553-551.

I2. English D], Bangdiwala SI, y Runyan DK: The dimensions of maltreatment: Introduction. Child Abuse \& Neglect 2005; 29: 44I-460.

13. Kinard EM: Methodological types of child maltreatment: Does the source of information make a difference? Child Abuse \& Neglect 1994; 18: 645-656.

14. Herrenkohl RC: The definition of child maltreatment: From case study to construct. Child Abuse \& Neglect 2005; 29: 4I3424.

15. Kairys SW, Johnson CF, Comitee on Child Abuse and Neglect: The psychological maltreatment of children-Technical report. Pediatrics 2002; 109: 68-70. http://www.pediatrics.org (visitado el 26 de Julio de 2006).

16. Jiménez Morago J, Moreno Rodríguez MC, Oliva Delgado A, Palacios González J, Saldaña Sage D: El maltrato infantil en Andalucía. Dirección General de Atención al Niño. Consejería de Trabajo y Asuntos Sociales. Junta de Andalucía. Sevilla, 1995. 17. Development and Psychopathology, 1991; 3: I-124.

18. American Professional Society on the Abuse of Children: Guidelines for psychological evaluation of suspected psychological maltreatment in children and adolescents. American Professional Society on the Abuse of Children. Chicago, 1995.

19. Steering Commitee for the Review of Commonwealth / State Service Provision: Efficiency Measures for Child Protection and Support Pathways: Reforms in Government Service Provision. Australia. Commonwealth of Australia. Camberra, 2003. at http://www.pc.gov.au/gsp/reports/reforms/emcpsp/emcpsp.pdf (visitado el 2 de octubre, 2006).

20. Creighton SJ: Introduction to Child Abuse Trends in England and Wales, 1988-1990: An Overview From 1973-1990. 1992. Citado por Loue S: Redefining the emotional and psychological abuse and maltreatment of children. Legal implications. Journal of Legal Medicine 2005; 26: 311-337.
21. O'Hagan KP: Emotional and Psychological Abuse: Problems of Definition, Child Abuse \& Neglect 1995; 19: 449-456.

22. Arruabarrena MI, de Paúl J: Maltrato a los niños en la familia. Evaluación y tratamiento. Pirámide. Madrid, 1994.

23. Straus MA, Kantor GK: Definition and measurement of neglectful behaviour: some principles and guidelines. Child Abuse \& Neglect 2005; 29: 19-29.

24. Hart S, Brassard M, Bingelli N, Davidson H: Psychological maltreatment. En Myers L, Berliner J, Briere C, Hendrix C, Jenny C, Reid T: The APSAC handbook on child maltreatment. Ia ed. Sage. Thousand Oaks, 2002. pp. 79-103.

25. Zuravin S: Child neglect: A review of definitions and measures research. En Dubowitz H: Neglected children: Research, practice and policy. Sage. Thousand Oaks, 1999. pp. 24-46.

26. Gaudin J: Child neglect: Short term and long term outcomes. En Dubowitz H: Neglected children: Research, practice and policy. Sage. Thousand Oaks, 1999. pp. 89-108.

27. Glaser D: Emotional abuse. En Hobbs C, Wynne J: Child abuse. Balliere Tindall. Londres, 1993. pp. 25I-267.

28. National Center of Child Abuse and Neglect: Child Maltreatment. National Center of Child Abuse and Neglect. Washington, 1997.

29. Doyle C: Emotional abuse of children: issues for intervention. Child Abuse review 1997; 6: 330-342.

30. Government Statistical Service: Children and young people on child protection registers. Departament of Health. Londres, 2000.

31. Inglés A: Els maltractaments infantils a Catalunya. Estudi global i balance de la seva situació actual. Generalitat de Catalunya. Barcelona, 1991.

32. Edis: El maltrato a menores en la ciudad de Zaragoza. Diputación General de Aragón, 1992. Documento no publicado, citado por Jiménez Morago J, Moreno Rodríguez MC, Oliva Delgado A, Palacios González J, Saldaña Sage D: El maltrato infantil en Andalucía. Dirección General de Atención al Niño. Consejería de Trabajo y Asuntos Sociales. Junta de Andalucía. Sevilla, 1995.

33. Simón Rueda C, López Taboada JL, Linaza Iglesias JL: Maltrato y desarrollo infantil. Universidad Pontificia de Comillas. Madrid, 2000.

34. Verdugo MA, Gutiérrez B, Fuertes J, Elices JA: Maltrato infantil y minusvalía. INSERSO. Madrid, 1993.

35. Garbarino J, Vondra J: Psychological maltreatment: issues and perspectives. En Brassard MR, Germain R, Hart SN: Psychological maltreatment of children and youth. Pergamon Press. New York, 1987. pp. 25-44.

36. Kelley JT: Stress and coping behaviours of substance abusing mothers. Journal of Social Pediatrics Nursing 1998; 3: 103-II0.

37. Hart SN, Brassard MR: A major threat to children's mental health. Psychological maltreatment. American Psychologist 1987; 42: 160-165.

38. Claussen AH, Crittenden P: Physical and psychological maltreatment: relations among types of maltreatment. Child Abuse \& Neglect 1991; 15: 5-18. 
39. Hart S, Brassard M, Karlson H: Psychological maltreatment. En: Berliner L, Briere J, Bulkley J, Jenny C, Reid T: The APSAC handbook on child abuse and neglect. Sage. Londres, 1996. pp. 72-89.

40. Sanders B, Becker-Lausen E: The measurement of psychological maltreatment: early data on the child abuse and trauma scale. Child Abuse \& Neglect 1995; 19: 315-323.

4I. Vissing YM, Straus MA, Gelles RJ, Harrop JW : Verbal aggression by parents and psychosocial problems of children. Child Abuse \& Neglect 1991; 5: 223-238.

42. Gómez de Terreros I, Gómez de Terreros M: Malos tratos infantiles. Aspectos psiconeurológicos. En: Gómez MR, Montilla Bono J, Nieto Barrera M: Neurología y Neuropsicología pediátrica. Diputación Provincial de Jaén. Jaén, 1995. pp.1519-1549.
43. Gómez de Terreros M: Consecuencias psicológicas y comportamentales. En: Gómez de Terreros I: Los profesionales de la salud ante el maltrato infantil. $2^{a}$ ed. Editorial Comares. Granada, 1997. pp. 210-216.

44. Huges HM, Graham-Bermann SA: Children of battered woman: impact of emotional abuse on adjustment and development. Journal of Emotional Abuse 1998; I: 23-50.

45. Citado por Loue S: Redefining the emotional and psychological abuse and maltreatment of children. Legal implications. Journal of Legal Medicine 2005; 26: 31I-337.

46. Christensen E: The Prevalence and Nature of Abuse and Neglect in Children Under Four: A National Survey, Child Abuse Review 1999; 8: 109-119. 